\section{Optical Negative Refraction in Bulk Metamaterials of Nanowires}

\author{
Jie Yao, ${ }^{1 *}$ Zhaowei Liu, $^{1 *}$ Yongmin Liu, ${ }^{1 *}$ Yuan Wang, $^{1}$ Cheng Sun, ${ }^{1}$ Guy Bartal, ${ }^{1}$ \\ Angelica M. Stacy, ${ }^{2}$ Xiang Zhang ${ }^{1,3} \dagger$
}

$\mathrm{M}$ etamaterials are artificially designed subwavelength composites possessing extraordinary optical properties that do not exist in nature. They can alter the propagation of electromagnetic waves, resulting in negative refraction (1), subwavelength imaging (2), and cloaking (3). First reported at microwave frequencies by using metamaterials made of an array of split ring resonators and metallic wires (4), negative refraction has been observed in two-dimensional (2D) photonic crystals into the infrared (IR) region (5-8) and in surface plasmon waveguides at visible frequencies (9). In both cases, negative refraction is constrained in two dimensions and is limited to a narrow band of frequencies. An indirect observation of negative refraction in the mid-IR region was also reported in a semiconductor multilayer structure (10). Creating bulk metamaterials that exhibit negative refraction for visible light remains a major challenge because of substantial resonance losses and fabrication difficulties. Recent theoretical studies suggest that metamaterials consisting of metal wire arrays exhibit an optical response at frequencies far away from resonances $(11,12)$, in which electromagnetic (EM) waves propagating along the nanowires exhibit negative refraction at a broad frequency band for all angles (13). Moreover, the material loss is much lower than traditional metamaterials with similar functionality.

We report observations of negative refraction in bulk metamaterials composed of silver nanowires with separation distance much smaller than the wavelength at optical frequencies (Fig. 1A). A porous alumina template was prepared by electrochemical anodization (14), into which silver nanowires were electrochemically deposited. A 1- $\mu \mathrm{m}$-wide slit, etched through a 250-nmthick silver film coated on the metamaterials, was illuminated by a collimated diode laser beam at different incident angles (see left side of Fig. 1A). The transmitted light was mapped by scanning a tapered optical fiber at the bottom surface of the metamaterial. The results are shown in Fig. 1, B and $\mathrm{C}$, for incident light at wavelengths of $660 \mathrm{~nm}$ and $780 \mathrm{~nm}$, respectively. When the incident angle is $30^{\circ}$, the transmitted beam is shifted to the left for transverse magnetic (TM)-polarized light, which corresponds to the negative refraction. The subwavelength composite forms an effective medium with opposite signs of electrical permittivities along and perpendicular to the wires (9). The hypobolic dispersion enables negative light refraction even though the phase velocity remains positive $(10,13,15)$. Conversely, the transverse electric (TE)-polarized light undergoes positive refraction. Figure 1D shows the dependence of refraction angles on a range of incident angles at $780 \mathrm{~nm}$. The group refractive indices of the metamaterial are shown to be -4.0 and 2.2 for TM and TE light, respectively. The phase
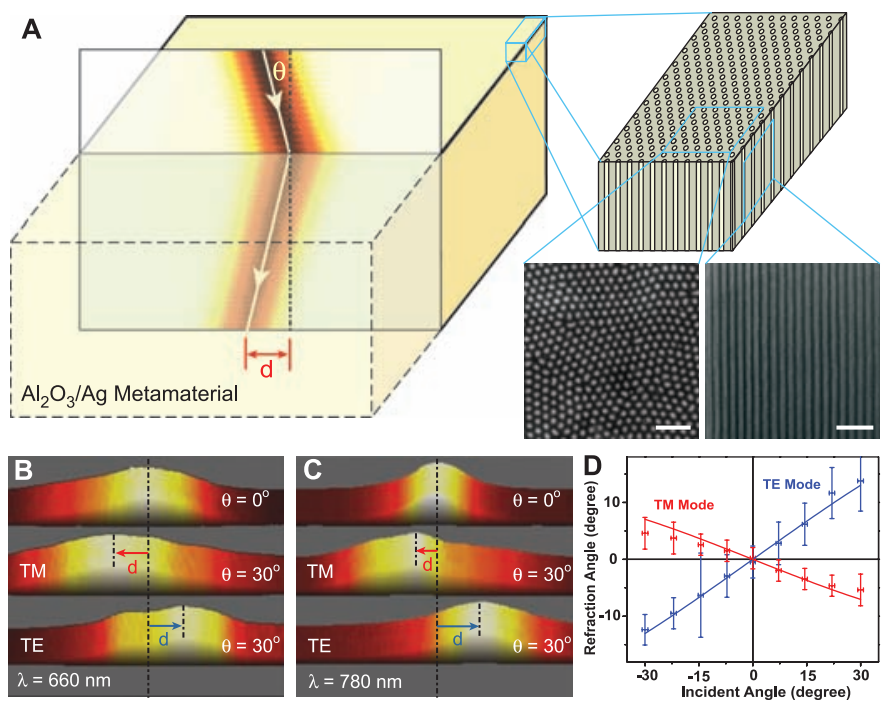

Fig. 1. Negative refraction in bulk metamaterial at visible frequencies. (A) (Left) Schematic of negative refraction from air into the silver nanowire metamaterials. (Right) Nanowires embedded in an alumina matrix, as well as scanning electron microscopy images showing the top and side view of the nanowires (60-nm wire diameter and 110-nm center-to-center distance). The scale bars indicate $500 \mathrm{~nm}$. Measured beam intensity at the existing surface of the metamaterial slab at the wavelength of $660 \mathrm{~nm}$ (B) and $780 \mathrm{~nm}$ (C). The lateral displacement (d) of TM polarized light shows the negative refraction in the metamaterial at both wavelengths, whereas TE light undergoes positive refraction. The horizontal sizes of (B) and (C) are $5 \mu \mathrm{m}$ and $12 \mu \mathrm{m}$, respectively. (D) The dependence of refraction angles on incident angles and polarizations at 780-nm wavelength. The negative refraction occurs for broad incident angles. The experiment data agree well with calculations (solid curves) using the effective medium theory. The sample thicknesses in (B) and (C) are $4.5 \mu \mathrm{m}$ and $11 \mu \mathrm{m}$, respectively. refractive index of the metamaterial remains positive, in contrast to that of left-handed metamaterials $(1,4)$. For normal incidence, the light intensity only decays $\sim 0.43 / \mu \mathrm{m}$ in the medium at 780 -nm wavelength, showing loss a few orders of magnitude lower than that of single-layer metamaterials reported at the same wavelength (16). Further calculations show that the negative refraction in this nanowire composite exists for longer wavelengths and also does not depend on the orientation of the nanowire lattice.

Because the dielectric response in this metamaterial does not require any resonance, the negative refraction has low loss and occurs in a broad spectral range, for all incident angles, making it an intrinsic optical response of the underlying metamaterials. Moreover, such bulk metamaterials can support propagating waves with large wave vectors that are evanescent in air or dielectrics, enabling manipulation of visible light at subwavelength scale. This can substantially affect applications such as waveguiding, imaging, and optical communication.

References and Notes

1. V. G. Veselago, Sov. Phys. Usp. 10, 509 (1968).

2. ]. B. Pendry, Phys. Rev. Lett. 85, 3966 (2000).

3. J. B. Pendry, D. Schurig, D. R. Smith, Science $\mathbf{3 1 2 ,}$ 1780 (2006); published online 24 May 2006 (10.1126/science.1125907).

4. R. A. Shelby, D. R. Smith, S. Schultz, Science 292, 77 (2001).

5. E. Cubukcu, K. Aydin, E. Ozbay, S. Foteinopoulou, C. M. Soukoulis, Nature 423, 604 (2003).

6. P. V. Parimi, W. T. Lu, P. Vodo, S. Sridhar, Nature 426, 404 (2003)

7. A. Berrier et al., Phys. Rev. Lett. 93, 073902 (2004).

8. E. Schonbrun et al., Appl. Phys. Lett. 90, 041113 (2007).

9. H. J. Lezec, J. A. Dionne, H. A. Atwater, Science 316, 430 (2007); published online 21 March 2007 (10.1126/science.1139266).

10. A. J. Hoffman et al., Nat. Mater. 6, 946 (2007).

11. R. Wangberg, ]. Elser, E. E. Narimanov, V. A. Podolskiy, J. Opt. Soc. Am. B 23, 498 (2006).

12. M. G. Silveirinha, P. A. Belov, C. R. Simovski, Phys. Rev. B 75, 035108 (2007).

13. More details are available as supporting material on Science Online.

14. H. Masuda, K. Fukuda, Science 268, 1466 (1995)

15. D. R. Smith, D. Schurig, Phys. Rev. Lett. 90, 077405 (2003).

16. G. Dolling, M. Wegener, C. M. Soukoulis, S. Linden, Opt. Lett. 32, 53 (2007).

17. This work was supported by the Air Force Office of Scientific Research Multidisciplinary University Research Initiative Program (grant no. FA9550-04-1-0434) and NSF NSEC under award no. DMI-0327077. The authors thank E. Hajime for assistance in fabrication at the early stage of the work.

\section{Supporting Online Material}

www.sciencemag.org/cgi/content/full/321/5891/930/DC1 Materials and Methods

Fig. S1

References

10 March 2008; accepted 4 June 2008

10.1126/science. 1157566

${ }^{1}$ National Science Foundation (NSF) Nanoscale Science and Engineering Center (NSEC), 5130 Etcheverry Hall, University of California, Berkeley, CA 94720-1740, USA. '2Department of Chemistry, University of California, Berkeley, CA 94720, USA. ${ }^{3}$ Materials Sciences Division, Lawrence Berkeley National Laboratory, 1 Cycletron Road, Berkeley, CA 94720, USA.

*These authors contributed equally to this work. †To whom correspondence should be addressed. E-mail: xiang@berkeley.edu 


\section{Science \\ \IAAAS}

\section{Supporting Online Material for}

\section{Optical Negative Refraction in Bulk Metamaterials of Nanowires}

Jie Yao, Zhaowei Liu, Yongmin Liu, Yuan Wang, Cheng Sun, Guy Bartal, Angelica M.

Stacy, Xiang Zhang*

*To whom correspondence should be addressed. E-mail: xiang@berkeley.edu

Published 15 August 2008, Science 321, 930 (2008)

DOI: 10.1126/science.1157566

\section{This PDF file includes:}

Materials and Methods

Fig. S1

References 


\section{Supporting Online Material}

\section{Optical Negative Refraction in Bulk Metamaterials of Nanowires}

Jie $\mathrm{Yao}^{1+}$, Zhaowei $\mathrm{Liu}^{1+}$, Yongmin $\mathrm{Liu}^{1+}$, Yuan $\mathrm{Wang}^{1}$, Cheng Sun ${ }^{1}$, Guy Bartal ${ }^{1}$, Angelica M. Stacy ${ }^{2}$, and Xiang Zhang ${ }^{1 *}$

${ }^{1}$ NSF Nano-scale Science and Engineering Center (NSEC), 5130 Etcheverry Hall, University of California, Berkeley, CA 94720-1740

${ }^{2}$ Department of Chemistry, University of California, Berkeley, California 94720

${ }^{+}$These authors contributed equally to this work

*To whom correspondence should be addressed. E-mail: xiang@berkeley.edu

\section{Materials and Methods}

\section{Sample fabrication}

The material was fabricated from porous alumina filled with silver nanowires. Polished and degreased high purity aluminum foil (99.9995\%, Alfa Aesar) was put into $0.2 \mathrm{M}$ oxalic acid for anodization. The anodized layer was then removed in a mixture of $\mathrm{H}_{3} \mathrm{PO}_{4}$ and $\mathrm{H}_{2} \mathrm{CrO}_{4}$. The first anodization left a regular array of dips on the metal surface, which were the starting points for the nanopores generated in the second anodization. Such a two-step anodization process assures excellent uniformity in terms of pore size and lattice distance in the final template. A silver seed layer was deposited (EB3, BOC Edwards) on the open side of the template. The barrier layer at the bottom of the template was then removed in diluted $\mathrm{H}_{3} \mathrm{PO}_{4}$. An electrochemical plating process was followed to grow silver nanowires inside the nanopores of the template. The overgrown nanowires on the top surface were removed by chemical mechanical polishing. Finally, a $1 \mu \mathrm{m}$ wide slit was etched through the silver seed layer using a Focused Ion Beam system (FIB, Strata 201XP, FEI).

\section{Material properties and numerical simulations}

At the long wavelength limit, the metamaterial with silver nanowires in an alumina matrix can be characterized as an effective uniaxial material $(S 1, S 2)$. The effective permittivity parallel to the nanowire is negative, and that perpendicular to the wire is positive over a very broad frequency region (from visible to near-infrared). The equifrequency contour (EFC) for a TM-polarized light is hyperbolic in the $k_{\mathrm{x}}, k_{\mathrm{z}}$ plane (S3), assuming the light propagation is along the wire (the z-axis). As shown in Figs. $\mathrm{S} 1(\mathrm{a})$, and (b), a TM wave incident from free space experiences negative refraction in the metamaterial although the phase front always undergoes positive refraction. 
(a)

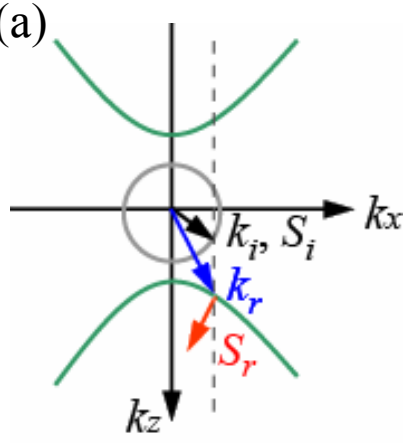

(b)

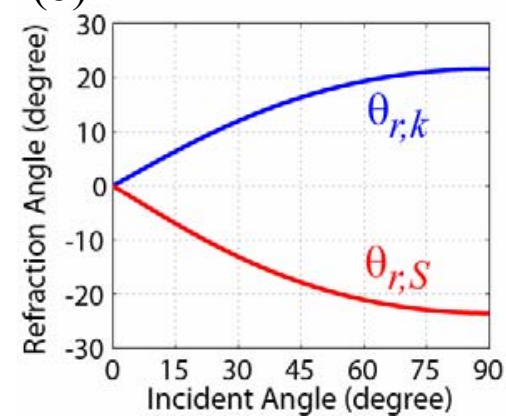

(c)

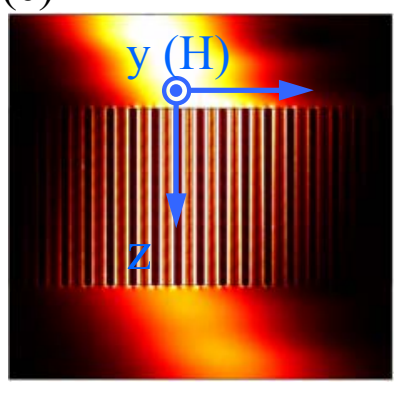

Fig. S1. (a) Schematic illustration of the negative refraction at the interface between the free space (circular gray EFC) and the metamaterial (green hyperbolic EFC). The Poynting vector is negatively refracted although the phase vector keeps positive. (b) The refraction angle in the metamaterial $\left(\varepsilon_{x}=5.28\right.$ and $\left.\varepsilon_{z}=-4.8 @ 660 \mathrm{~nm}\right)$ versus the incident angle from air. $\theta_{r, k}$ and $\theta_{r, S}$ represent refraction angles for the wave vector and Poynting vector, respectively. (c) One exampled numerical demonstration of negative refraction in such metamaterials.

We performed full-wave simulations (by COMSOL Multiphyiscs ${ }^{\mathrm{TM}} 3.4$,) considering the typical nanowire dimensions in our sample and real material permittivies for $\mathrm{Al}_{2} \mathrm{O}_{3}\left(\varepsilon_{\mathrm{Al} 2 \mathrm{O} 3}=2.4\right)$ and $\mathrm{Ag}\left(\varepsilon_{\mathrm{Ag}}=-21.6+0.8 \mathrm{i}\right)(\mathrm{S} 4)$ at the wavelength of $660 \mathrm{~nm}$. The incident angle is 30 degrees and the nanowire length is $1.5 \mathrm{um}$. The negative refraction is clearly seen as shown in Fig. S1(c) and agrees well with the prediction based on the effective media theory (Fig. S1(b)). Negative refraction was also obtained in the same sample at NIR frequencies (simulation data not shown here).

\section{Optical measurements}

The sample with the slit was illuminated by a diode laser at $660 \mathrm{~nm}$ and $780 \mathrm{~nm}$ (LTC78025AH, LTC66012AH, Lasermate), and the transmitted light was mapped by scanning a tapered optical fiber at the exit surface. The incident angle of the light was controlled by a stage and the polarization can be selected by a linear polarizer. We marked the center position of the transmitted beam under normal illumination as a reference. The lateral displacement of the beam under a given incident angle and polarization was determined by comparing the center position of the beam with respect to the reference.

\section{Reference:}

S1. G. W. Milton, The Theory of Composites, Cambridge University Press, (2002)

S2. R. Wangberg, J. Elser, E. E. Narimanov, V. A. Podolskiy, J. Opt. Soc. Am. B 23, 498 (2006)

S3. P. A. Belov, Microwave Opt. Technol. Lett. 37, 259 (2003)

S4. P. B. Johnson, R. W. Christy, Phys. Rev. B 6, 4370 (1972); C. A. Foss, G. L. Hornyak, J. A. Stockert, and C. R. Martin, J. Phys. Chem, 98, 2963 (1994) 